\title{
ОПРЕДЕЛЕНИЕ ПОЛОМОК РЕЗЦОВ РОС С ПОМОЩЬЮ РЕГРЕССИОННОГО И НЕЙРОСЕТЕВОГО МОДЕЛИРОВАНИЯ
}

\author{
Третьяк Александр яковлевич', \\ 13050465@mail.ru
}

Кузнецова Алла Витальевна', alvitkuz@yandex.ru

\author{
Борисов Константин Андреевич', \\ 13020165@mail.ru
Южно-Российский государственный политехнический университет (НПИ) им. М.И. Платова, Россия, 346428, г. Новочеркасск, ул. Просвещения, 132.

\begin{abstract}
Актуальность. В процессе бурения скважин буровые долота, армированные пластинами PDC, отрабатывают свой ресурс не полностью, так как часть режущих PDC элементов выходит из строя по причине их скола, слома или потери, что в значительной степени сказывается на конечных технико-экономических показателях бурения скважин.

Цель: разработка и предложение нейросетевой модели для решения проблемы определения процента поломок режущих элементов буровых долот, армированных PDC, на основе имеющихся полевых данных бурения скважин по породам VI-VIII категории по буримости.

Объекты: причины, вызывающие выход из строя пластин PDC на буровых долотах в процессе бурения скважин в породах VI-VIII категории по буримости в зависимости от режимных параметров бурения.

Методы. В качестве факторов для определения процента поломок использовалась нагрузка на долото (Р, кН), частота его вращения (N, об/мин) и скорость бурения ( $V, M / 4)$ при неизменных значениях параметров промывочной жидкости и компоновки низа бурильной колонны. Для анализа данных применялись регрессионные и нейросетевые модели различных конфигураций. Результаты. Анализ регрессионных моделей показал их непригодность из-за нелинейного характера скорости бурения при низких давлениях и высоких оборотах. Авторами предложена двухступенчатая нейросетевая модель, в которой первая нейронная сеть (Neural Network) служит для определения скорости бурения, а вторая - для прогнозирования процента поломок пластин PDC. Ошибки нейросетевого ансамбля на тестовых данных не превышают величину пригодности нелинейных моделей, равную 4,5 \% для относительной ошибки и 12-15 \% для максимальной величины. Предложенная нейросетевая модель может найти применение при разработке технологического регламента отработки долот, армированных резцами PDC.
\end{abstract}

\section{Ключевые слова:}

Поломки PDC, искусственная нейронная сеть, регрессионная модель, технологический регламент отработки долот, торсионные вибрации.

\section{Введение}

Бурение скважин на нефть и газ в комплексе работ при геологоразведке выходит на первое место как по стоимости работ, так и по качеству и количеству геологической информации, получаемой в ходе их проведения. Породоразрушающим инструментом бурят осадочные, метаморфические и магматические породы на глубину до семи километров. Эффективность процесса бурения скважин, особенно в твердых горных породах, в значительной степени определяется эксплуатационными показателями породоразрушающего инструмента [1-4].

В последнее время на первый план выходит породоразрушающий инструмент, армированный алмазно-твердосплавными пластинами (АТП) или Polycrystalline diamond compact (PDC) [5-9]. B paботе сделана попытка обобщения и систематизации результатов исследований по технологии износа и поломок буровых долот, армированных PDC.

Известно, что вибрации бурового долота, армированного $\mathrm{PDC}$, являются основной причиной износа алмазных резцов. На долото могут действовать различные типы вибраций: осевая, радиальная (поперечная), торсионная (эффект пружины), но радиальная и торсионная вибрации встречаются наиболее часто (рис. 1).

Осевая вибрация зависит от нагрузки и частоты вращения долота, а также жесткости компоновки низа бурильной колонны (КНБК). Торсионные колебания вызывают снижение скорости вращения КНБК при внедрении в горную породу на забое, вплоть до остановки вращения. При этом энергия вращения непрерывно сообщается долоту буровым станком по колоннам бурильных труб или забойными двигателями до тех пор, пока она не превысит предел прочности породы. В это время буровой снаряд с породоразрушающим инструментом (ПРИ) начинает раскручиваться с ускорением, превышающим в два или более раза номинальную скорость вращения. При этом, как и при поперечной вибрации, увеличивается ударная нагрузка на резцы PDC, что может привести к их сколу, слому и потере, в зависимости от глубины внедрения резца в породу и его положения на забое.

Торсионные колебания зависят от глубины внедрения PDC в горную породу. При большой глубине внедрения реактивный момент возрастает, а, следовательно, возрастает и риск возникновения 
торсионных колебаний. При использовании PDC меньшего диаметра глубина внедрения резцов будет меньше, что способствует снижению реактивного момента. Справиться с влиянием данного эффекта можно уменьшением скорости вращения ПРИ и увеличением осевой нагрузки на режущие PDC элементы. Радиальная вибрация - это такой тип вибрации, при котором вибрация долота происходит вокруг его оси и не является его геометрическим центром. Происходит быстрое ускорение и замедление вращения долота, что значительно увеличивает ударную нагрузку на PDC. Добиться уменьшения радиальной вибрации можно путем увеличения нагрузки на PDC, уменьшением частоты вращения и увеличения жесткости КНБК [10-12].

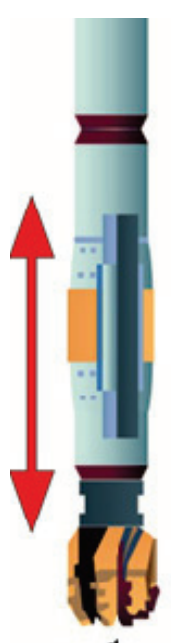

1

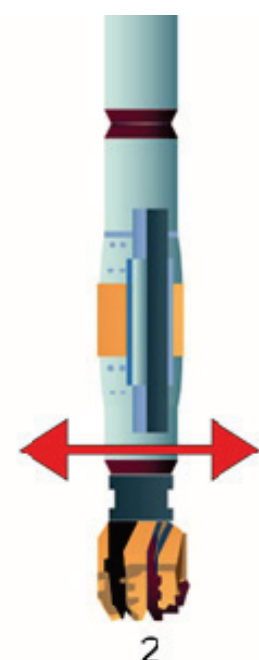

2

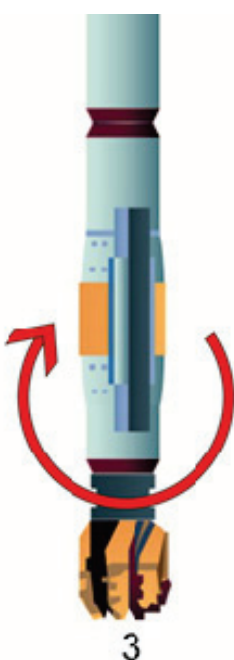

3
Pис.1. Основные виды вибраций долота, армированного РDC, при бурении скважин: 1) осевая; 2 ) радиальная (поперечная); 3 ) торсионная (эффект пружины)

Fig. 1. Main types of vibration bit, reinforced $P D C$, when drilling wells: 1) axial; 2) radial (transverse); 3) torsional (spring effect)

\section{Задача}

Опыт эксплуатации буровых долот, армированных PDC, показывает, что наибольший процент поломок от вибраций (более 50 \%) происходит по причине поломок резца, то есть скола и слома. Исходя из вышеизложенного, была поставлена задача - минимизировать количество поломок резцов PDC на буровом долоте. Решение этой задачи будет способствовать увеличению проходки на долото и механической скорости бурения.

Поломка PDC - есть результат частичного выхода из строя долота при неудачно выбранных значениях основных параметров бурения - нагрузки Р и частоты вращения N. Оптимизируя эти два основных технологических параметра, при оптимально заданных жесткости низа буровой колонны и количеству прокачиваемой жидкости, необходимо добиться минимизации количества поломок резцов PDC бурового долота.
Для буровых компаний очень важно добиться оптимальной технологии отработки долот, армированных PDC. В распоряжении технолога-буровика не всегда имеются в наличии сведения обо всех возможных поломках резцов породоразрушающего инструмента и о возможных причинах их появления, возникающих при бурении или в нештатных ситуациях. Поэтому обобщить знания специалиста на все возможные диапазоны параметров бурения можно как на основе математикостатистических методов регрессионного анализа, так и на основе популярных в настоящее время технологий искусственного интеллекта и, в частности, искусственных нейронных сетей (ИНС).

Поломки резцов PDC в процессе бурения скважин были всегда и от них невозможно избавиться полностью, но, подбирая оптимальное значение $P$ и $N$ и получая при этом максимальную механическую скорость бурения $V$ как определяющий фактор, peшим задачу минимизации поломок резцов PDC.

\section{Решение}

Исследования проводились с использованием полевых данных обработки долот, армированных PDC, диаметром 215,9 мм, с 8 лопастями, вооруженными 56 пластинами PDC. Бурение скважин осуществлялось по горным породам VI-VIII категории по буримости. Фрагмент исходных данных для создания моделей представлен в табл. 1.

Полный выход долота из строя - редкость, а процент частичной поломки резцов PDC эксперт определяет следующим образом:

$$
\text { Prpol }=\left(n_{\text {пол }} / n_{\text {общ }}\right) \cdot 100 \%,
$$

где $n_{\text {пол }}$ - число резцов $\mathrm{PDC}$, вышедших из строя за фиксированный момент времени; $n_{\text {общ }}$ - общее число элементов долота.

Анализ имеющихся экспериментальных данных показал низкую линейную корреляционную зависимость между $P$ и $N\left(R_{P, N}=0\right)$, что является условием включения этих параметров в создаваемую модель в качестве факторных.

Высокая зависимость между скоростью и процентом поломок $\left(R_{V, \text { Prpol }}=0,79\right)$ позволяет рассматривать их в качестве результативных параметров, либо один из них может быть входным для другого. Линейные корреляционные зависимости входных и выходных параметров представлены в табл. 2 .

Низкая связь между $N$ и $V, N$ и $\operatorname{Prpol}\left(R_{N, V}=0,05\right.$; $\left.R_{N, P r p o l}=0,04\right)$ свидетельствует о том, что прямой линейной зависимости между этими парами параметров нет. Но это совсем не значит, что между ними отсутствуют другие, нелинейные зависимости. По мнению авторов, влияние частоты оборотов на рассматриваемые выходные параметры бурения является существенно нелинейной. Косвенно это подтверждается непригодностью созданных линейных регрессионных моделей, построенных при уровне доверительного интервала 0,05 :

$$
\begin{gathered}
V_{\text {reg }}=-0,06+0,49 P+0,00 N ; \\
\text { Prpol }_{\text {reg }}=-10,49+0,97 P+0,00 N .
\end{gathered}
$$


Таблица 1. Фрагмент обучающей выборки

Table 1. Fragment of the training sample

\begin{tabular}{|c|c|c|c|c|}
\hline $\begin{array}{c}\text { Скорость бурения } \\
\text { Drilling speed }\end{array}$ & $P, \mathrm{kH}$ & $\begin{array}{c}N, \text { об/мин } \\
(\mathrm{r} / \mathrm{min})\end{array}$ & $\begin{array}{l}V, \mathrm{M} / \mathrm{ч} \\
(\mathrm{m} / \mathrm{h})\end{array}$ & $\begin{array}{c}\text { PrPolom, } \\
\%\end{array}$ \\
\hline \multirow{11}{*}{ Низкая/Low } & 7 & 200 & 2,0 & 0,5 \\
\hline & 7 & 210 & 2,2 & 0,5 \\
\hline & 8 & 230 & 3,2 & 0,6 \\
\hline & 8 & 240 & 3,5 & 0,6 \\
\hline & 8 & 250 & 4,0 & 0,6 \\
\hline & 9 & 260 & 4,2 & 0,7 \\
\hline & 9 & 270 & 4,7 & 0,7 \\
\hline & 9 & 280 & 5,0 & 0,7 \\
\hline & 10 & 290 & 5,6 & 0,7 \\
\hline & 10 & 300 & 5,9 & 0,7 \\
\hline & 10 & 310 & 6,0 & 0,8 \\
\hline \multirow{14}{*}{ Средняя/Average } & 13 & 240 & 7,4 & 1,6 \\
\hline & 13 & 250 & 7,6 & 1,7 \\
\hline & 13,5 & 260 & 7,8 & 1,7 \\
\hline & 13,5 & 270 & 7,9 & 1,8 \\
\hline & 13,5 & 280 & 7,9 & 1,9 \\
\hline & 14 & 290 & 8,0 & 1,9 \\
\hline & 14 & 300 & 8,0 & 2,0 \\
\hline & 14 & 310 & 8,1 & 2,1 \\
\hline & 14,5 & 200 & 8,1 & 2,2 \\
\hline & 14,5 & 210 & 8,2 & 2,6 \\
\hline & 14,5 & 220 & 8,3 & 3,4 \\
\hline & 15 & 230 & 8,4 & 4,3 \\
\hline & 15 & 240 & 8,4 & 4,5 \\
\hline & 15 & 250 & 8,4 & 4,7 \\
\hline \multirow{11}{*}{ Высокая/High } & 18,5 & 210 & 9,2 & 9,3 \\
\hline & 18,5 & 220 & 9,2 & 9,4 \\
\hline & 19 & 230 & 9,3 & 9,6 \\
\hline & 19 & 240 & 9,3 & 9,7 \\
\hline & 19 & 250 & 9,4 & 9,7 \\
\hline & 19,5 & 260 & 9,6 & 10,5 \\
\hline & 19,5 & 270 & 9,7 & 10,6 \\
\hline & 19,5 & 280 & 9,8 & 10,8 \\
\hline & 20 & 290 & 10,0 & 14,9 \\
\hline & 20 & 300 & 10,3 & 15,0 \\
\hline & 20 & 310 & 10,5 & 15,1 \\
\hline
\end{tabular}

Таблица 2. Коэффициенты корреляиии паралетров модели

Table 2. Correlation coefficients of model parameters

\begin{tabular}{|c|c|c|c|c|}
\hline & $P$ & $N$ & $V$ & Prpol \\
\hline$P$ & 1 & 0 & 0,95 & 0,93 \\
\hline$N$ & 0 & 1 & 0,05 & 0,04 \\
\hline$V$ & 0,95 & 0,05 & 1 & 0,79 \\
\hline Prpol & 0,93 & 0,04 & 0,79 & 1 \\
\hline
\end{tabular}

Коэффициенты регрессии для частоты вращения $\mathrm{N}$ в моделях (1) и (2) имеют порядок значений $10^{-4}(0,0029$ и 0,0046$)$, что практически исключает её из рассмотрения в качестве основного параметра регрессионной модели. Средние и максимальные значения ошибок моделей соответственно равны 8,6 и $96,4 \%$ для (1), 82,1 и $323 \%$ для (2), что свидетельствует об их полной непригодности.

Создание модели для вычисления процента поломок на основе трех параметров $P, N$ и $V$ :

$$
\text { Prpol }_{\text {reg }}=-10,63+1,96 P+0,01 N-2,02 \mathrm{~V}
$$

ненамного улучшило ситуацию по сравнению с линейной моделью (2). Величины ошибок модели (3) соответственно равны 35,7 и $214 \%$. Справедливости ради следует отметить, что выборы в значениях ошибок регрессионных моделей наблюдаются в области низких давлений (там имеют место существенные нелинейные зависимости) и частично в области высоких давлений.

Попытка создания нелинейных регрессионных моделей $(4,5)$ на основе метода наименьших квадратов (МНК) с использованием метода Гаусса-Ньютона и последующей проверкой применимости МНК показала следующие результаты:

$$
V_{\text {nlreg }}=-0,04 P^{2}+0,00 N^{2}+1,53 P+0,00 T-6,62 ;
$$

Prpol $_{\text {nlreg }}=-0,10 P^{2}-0,00 N^{2}-1,82 P+0,01 T+7,21$. (5)

Уровень значимости $\alpha$ в обоих случаях был выбран равным 0,05. Проверка истинности пяти предпосылок МНК [13], а именно: 1) случайный характер остатков; 2) нулевая средняя величина остатков; 3) нормальное распределение остатков; 4) отсутствие автокорреляции остатков; 5) постоянство дисперсии остатков (гомоскедастичность) дала положительный ответ для предпосылок № 1-3. Выявлено наличие тенденции при проверке предпосылки № 4. Зарегистрирован незначительный выход за пределы доверительного интервала крайних левых (низкие давления) и крайних правых (высокие давления) значений вектора остатков при проверке гомоскедастичности. Величины средних и максимальных значений ошибок 9,1 и 24,6 \% для модели (4); 19,4 и 65,6 \% для модели (5) все же превышают максимально допустимое значение пригодности нелинейных моделей, равное $12-15 \%$.

Неполное удовлетворение предпосылок МНК и превышение порога пригодности требуют либо существенной корректировки рассматриваемых нелинейных моделей либо использование принципиально иных методов и технологий определения оптимальных значений выходных параметров - аппарата теории искусственных нейронных сетей. Способность к обобщению информации, описывающей нелинейные зависимости в сложных объектах, процессах и явлениях, неотъемлемая черта ИНС, присущая ей по внутренней природе по структуре и по способу функционирования на основе обучения. Современный уровень технологии буровых работ как раз и относится к подобным высокотехнологичным наукоёмким процессам, характеризующимся большим числом режимов, типов параметров, объемов характеристик каждого типа, связанных сложными нелинейными соотношениями.

Нейронные сети помогают выявить связи между экспериментальными данными в тех случаях, когда статистические методы не справляются с задачей. По сравнению со статистико-математическими методами, ИНС менее требовательны к качеству априорных знаний. В рассматриваемом случае в качестве таких знаний выступает ограниченный состав выбранных входных параметров нейросетевой модели, отсутствие достоверных производ- 
ственных данных для большинства граничных и ряда средних значений входных параметров. В подобных условиях нейронные сети часто дают быстрое и качественное решение задачи, как правило, не худшее, чем решение, получаемое статистическими методами, после тщательной подготовки данных для обучения сети. Разработка промышленно пригодной нейросетевой системы для определения управляющих параметров технологических процессов, как правило, осуществляется за значительно более короткое время (порядка нескольких месяцев) по сравнению с созданием аналогичной системы, требующей тщательного выбора математической модели на основе анализа имеющихся производственных данных, и информации, специфичной для данной области [15-20].

Для построения модели была использована нейронная сеть прямого распространения сигнала (feedforward) с двумя входами и двумя выходами (пакет NeuralNetworks системы Matlab). В результате циклического поиска наилучшей, в смысле минимума относительной ошибки на обучающей и тестовой выборке, оказалась сеть с двумя скрытыми слоями с десятью и пятью нейронами (рис. 2).

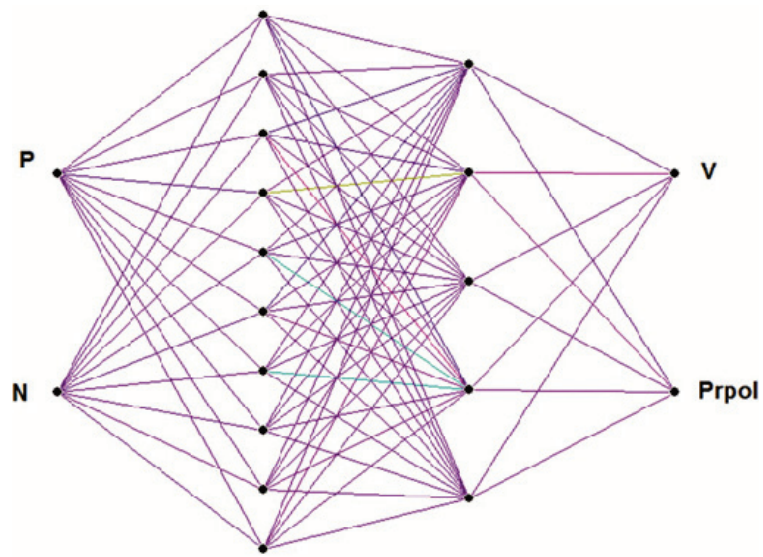

Puc. 2. Нейронная сеть для определения V, Prpol на основе $P, N$

Fig. 2. Neural network to determine V, Prpol on the basis of $P, N$

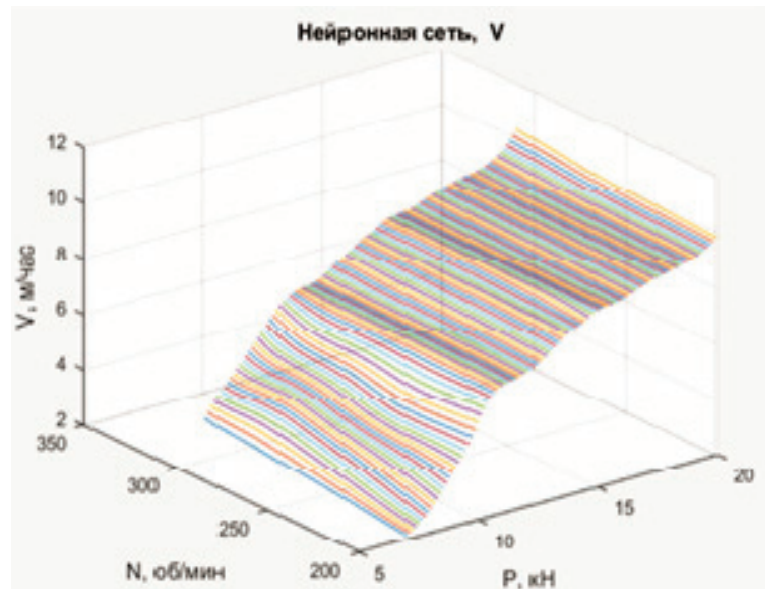

Функция обучения нейронов скрытого слоя гиперболический тангенс (tansig), выходного слоя - линейная (purelin), алгоритм обучения Левенберга-Марквардта. Функция вычисления ошибки при обучении сети среднеквадратическая (mse). Способ выбора элементов обучающей выборки в процессе обучения случайный. Остальные параметры обучения сети выбраны по умолчанию. Для обучения нейронной сети использовались те же данные, что и при составлении регрессионных моделей. Из общего количества входных векторов было выделено тестовое множество в объёме $20 \%$ от общего числа векторов. Поскольку диапазоны входных и выходных параметров различны, их значения параметров были линейно масштабированы по диапазону изменения в интервал [0..1]:

$$
\tilde{x}=\frac{\left(x_{i}-x_{\min }\right)}{\left(x_{\max }-x_{\min }\right)},
$$

где $\tilde{x}$ - масштабированное значение параметра; $x_{i}-$ текущее значение параметра; $x_{\min }, x_{\max }$ - минимальное и максимальное значения параметра.

Абсолютные и относительные величины ошибок оптимальной сети оказались гораздо меньше величин ошибок, которые демонстрировали регрессионные модели. Анализ остатков, полученных в результате тестирования нейросетевой модели, показал, что, несмотря на достаточно высокую среднюю величину этих остатков по сравнению с регрессионной моделью $\left(10^{-2}, 10^{-3}\right.$ против $\left.10^{-14}\right)$, остальные характеристики полностью удовлетворяют предпосылкам № 1-5. Этот факт, по мнению авторов, свидетельствует в пользу применения ИНС-модели, учитывающей нелинейные зависимости.

Множество значений скоростей и процента поломок, вычисляемое нейронной сетью на всем диапазоне изменения давления $7 . .20 \mathrm{\kappa H}$ и частоты вращения 200...310 об/мин, представлено на рис. 3.

Несмотря на приемлемые величины средних ошибок сети, максимальные значения ошибок нейросетевой модели всё еще значительны. Боль-

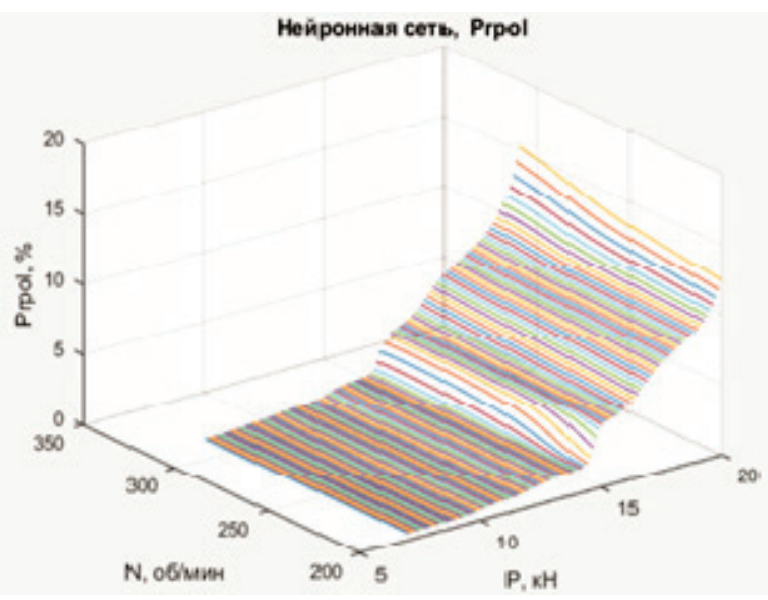

Pис. 3. Реакиия сети на входные воздействия: слева - скорость бурения V; справа - процент поломок Prроl

Fig. 3. Network reaction to the input: on the left is the drilling speed $V$; to the right is the percentage of breakdowns Prpol 


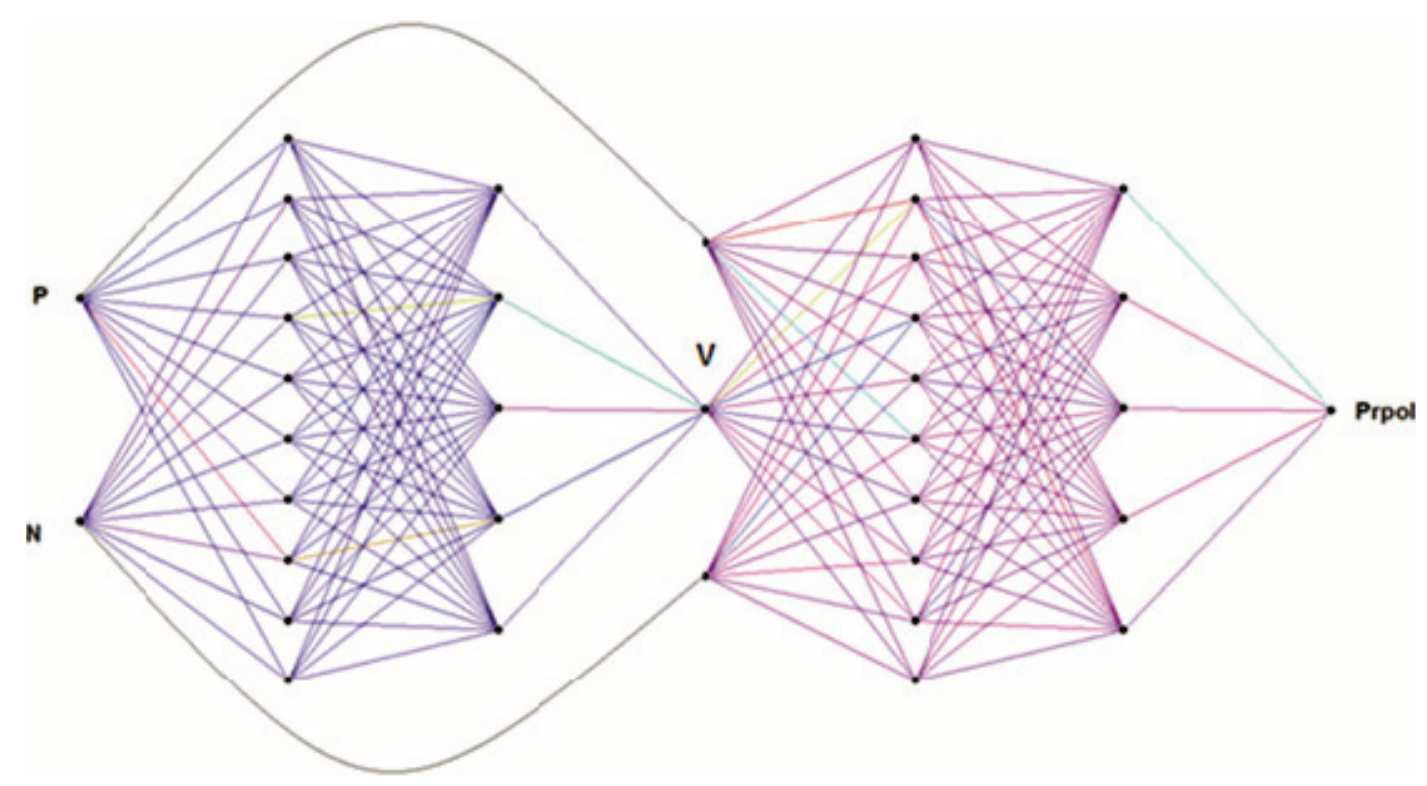

Рис. 4. Ансамбль нейронных сетей

Fig. 4. Ensemble of neural networks

шая часть выбросов наблюдалась в области низких давлений, реже - в области высокого давления и почти никогда при средних значениях давления. Учитывая вышесказанное, а также то, что механическая скорость бурения является определяющим фактором при выбраковке резцов PDC, предложенная модель вида:

$$
\{P, N\} \rightarrow\{N N: 10 \times 5\} \rightarrow\{V, \text { Prpol }\}
$$

была преобразована в новую модель, состоящую из двух последовательно соединённых сетей:

$$
\begin{gathered}
\{P, N\} \rightarrow\{N N: 10 \times 5\} \rightarrow\left\{V^{*}\right\} ; \\
\left\{P, N, V^{*}\right\} \rightarrow\{N N: 10 \times 5\} \rightarrow\{\text { Prpol }\} .
\end{gathered}
$$

Первая нейронная сеть ансамбля вычисляет значение скорости бурения $V^{*}$, а вторая сеть использует полученную скорость в совокупности с давлением и частотой оборотов долота для вычисления процента поломок (рис. 4).

В этом случае не только средние значения ошибок обеих сетей, но и выбросы ошибок снижены до приемлемого уровня 12-15\%. Величины ошибок, при вычислении процентов поломок двумя предложенными моделями (6) и (7), представлены в табл. 3.

На рис. 5 приведены поверхности отклика модели (7) при низких (200), средних (250) и высоких (310) значениях частоты оборотов. Результаты экспериментов показывают, что последовательно соединенные сети моделируют сложные поверхности для вычисляемых значений $V$ и $P r p o l$ в области известных значений входных параметров $P, N$ в отличие от более «гладких» поверхностей регрессионных моделей. С одной стороны, это указывает на более тонкую настройку модели, способной точнее определять значения искомых величин, с другой - на возможную подстройку сетей под данные обучающей выборки, сформированной экспертом.

\begin{tabular}{|c|c|c|c|c|}
\hline \multirow{2}{*}{$\begin{array}{l}\text { Ошибки нейронной сети } \\
\text { Errors of the neural network }\end{array}$} & \multicolumn{2}{|c|}{$\begin{array}{c}\text { Модель } 1 \\
\text { Model } 1\end{array}$} & \multicolumn{2}{|c|}{$\begin{array}{c}\text { Модель } 2 \\
\text { Model } 2\end{array}$} \\
\hline & V & Prpol & V & Prpol \\
\hline $\begin{array}{l}\text { Среднее значение остатков } \\
\text { на обучающей выборке } \\
\text { Average value of residuals } \\
\text { in the training sample } \\
\end{array}$ & $-0,02$ & $-0,01$ & $-0,01$ & $-0,01$ \\
\hline $\begin{array}{l}\text { Среднее значение остатков } \\
\text { на тестовом множестве } \\
\text { Average value of residuals on the test set }\end{array}$ & $-0,01$ & $-0,03$ & $-0,01$ & $-0,01$ \\
\hline $\begin{array}{l}\text { Среднее значение относительной ошибки } \\
\text { на обучающей выборке } \\
\text { Average value of the relative error } \\
\text { in the training sample }\end{array}$ & 1,29 & 3,58 & 2,74 & 2,76 \\
\hline $\begin{array}{l}\text { Среднее значение относительной ошибки } \\
\text { на тестовом множестве } \\
\text { Average value of the relative error } \\
\text { on the test set }\end{array}$ & 2,05 & 4,48 & 2,95 & 2,82 \\
\hline $\begin{array}{l}\text { Максимальное значение относительной } \\
\text { ошибки на обучающей выборке } \\
\text { Maximum value of the relative error } \\
\text { in the training sample }\end{array}$ & 12,82 & 19,90 & 10,67 & 11,96 \\
\hline $\begin{array}{l}\text { Максимальное значение относительной } \\
\text { ошибки на тестовом множестве } \\
\text { Maximum value of the relative error } \\
\text { on the test set }\end{array}$ & 14,74 & 16,89 & 11,03 & 13,07 \\
\hline
\end{tabular}

Таблица 3. Величины ошибок нейронных сетей

Table 3. $\quad$ Error values of neural networks

\section{Выводы}

1. Использование нейросетевых моделей для целей минимизации поломок резцов $\mathrm{PDC}$ является актуальной задачей.

2. Предложенная модель ансамбля нейронных сетей позволяет добиться увеличения механической скорости бурения скважин на нефть и газ, а также проходку на долото, с учетом уменьшения поломок PDC. 


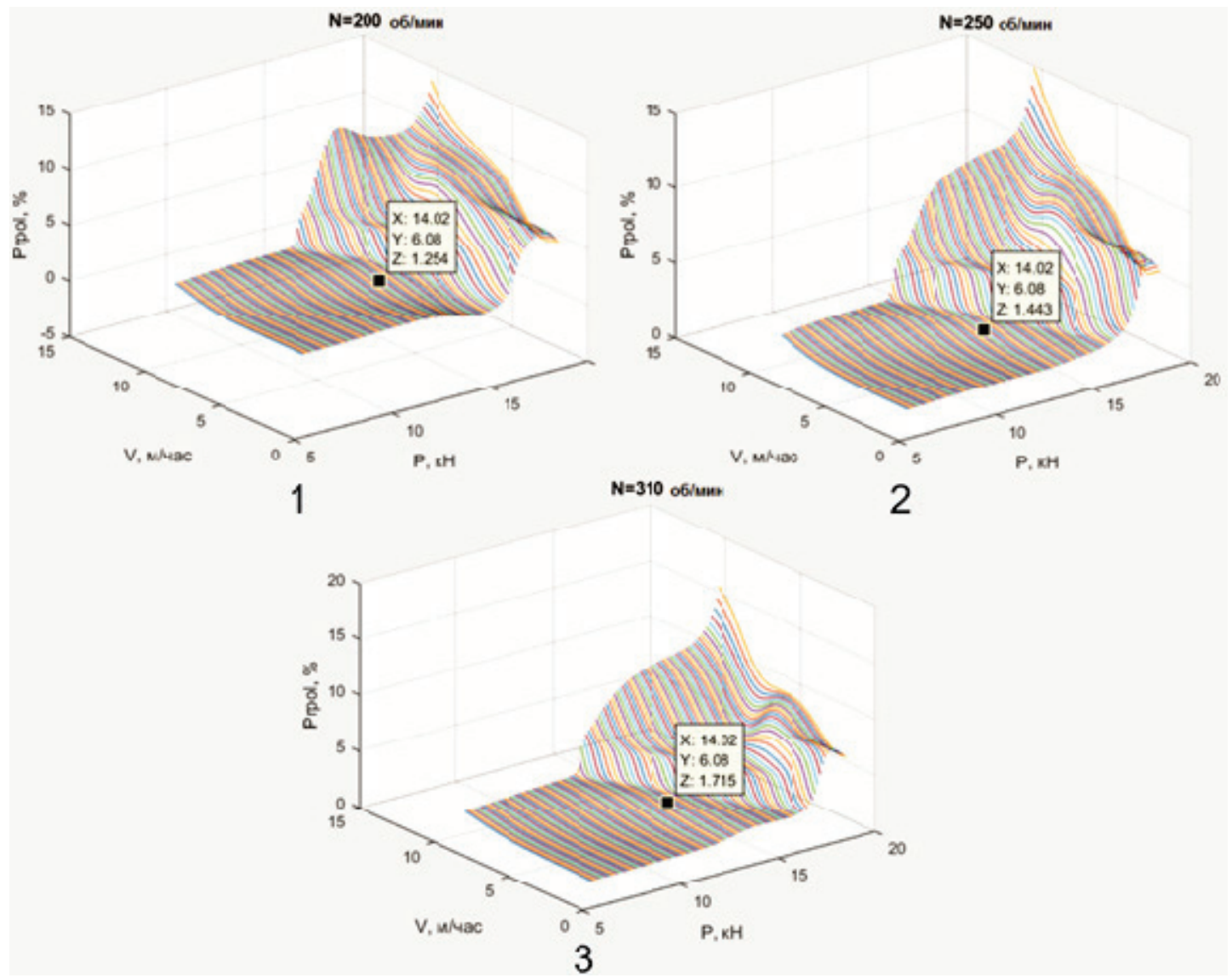

Puс. 5. Реакиия второй сети (процент пололок): 1) $N=200 ; 2$ ) $N=250 ; 3$ ) $N=310$

Fig. 5. Reaction of the second network (breakdowns percentage): 1) $N=200 ; 2$ ) $N=250 ; 3$ ) $N=310$

3. Модель может быть использована при оперативном учете основных параметров бурения в системе геолого-технологических исследований.

4. Положительные результаты, полученные с помощью комплексной нейросетевой модели с использованием полевых данных отработки долот, армированных PDC, показали её пригодность и возможность дальнейшего развития путем учёта дополнительных параметров режима бурения и уточнения уже использованной входной информации.

\section{СПИСОК ЛИТЕРАТУРЫ}

1. Инновационные подходы к конструированию высокоэффективного породоразрушающего инструмента / А.Я. Третьяк, В.В. Попов, А.Н. Гроссу, К.А. Борисов // ГИАБ. - 2017. № 8. - C. $225-230$.

2. Метод расчета осевой нагрузки и механической скорости бурения двухъярусного долота режущего типа Д-2ВВ / А.Я. Третьяк, В.Ф. Чихоткин, Ю.Ф. Литкевич, А.Е. Асеева / Строительство нефтяных и газовых скважин на суше и на море. 2006. - № 3. - C. 13-18.

3. Нескоромных В.В., Пушмин П.С. Методика оптимальных параметров режима и условий бурения скважин // Известия Сибирского отделения Секции наук о Земле РАЕН. - 2011. № 1 (38). - C. 151-157.
5. Предложенная нейросетевая модель и её последующие модификации могут найти широкое применение при разработке технологического регламента отработки долот, армированных резцами $\mathrm{PDC}$.

6. Использование нейросетевых моделей для целей оптимизации техники и технологии сооружения скважин на нефть и газ в настоящее время востребовано производственниками и требует дальнейшего совершенствования.

4. Нескоромных В.В., Борисов К.И. Аналитическое исследование процесса резания-скалывания горной породы долотом с резцами $\mathrm{PDC} / /$ Известия Томского политехнического университета. - 2013. - Т. 323. - № 1. - С. 191-195.

5. Третьяк А.А., Литкевич Ю.Ф., Асеева А.Е. Разработка методики расчёта наработки породоразрушающего инструмента с алмазно-твердосплавным вооружением // Строительство нефтяных и газовых скважин на суше и на море. - 2010. - № 12. C. 2-5.

6. Третьяк А.А. Влияние вибраций на прочностные свойства буровых коронок, армированных алмазно-твердосплавными пластинами // Строительство нефтяных и газовых скважин на суше и на море. - 2016. - № 10. - С. 20-24.

7. Сысоев Н.И., Буренков Н.Н., Третьяк А.А. Расчет конструк- 
тивных параметров буровых коронок, армированных PDC // Oil and Gas Journal Russia. - 2012. - № 5. - C. 56-58.

8. Симонов В.В., Юнин Е.К. Влияние колебательных процессов на работу бурильного инструмента. - М.: Недра, 1977. - 210 с.

9. Власюк В.И., Будюков Ю.Е., Спирин В.И. Технические средства и технологии для повышения качества бурения скважин. - Тула: Гриф и К, 2013. - 176 с.

10. Третьяк А.А., Савенок О.В., Швец В.В. Буровые коронки, армированные алмазно-твердосплавными пластинами. - Новочеркасск: Изд-во Южно-Российского государственного политехнического университета (НПИ), 2015. - 186 с.

11. Ресурсосберегающая технология алмазного бурения в сложных геологических условиях / Н.В. Соловьев, В.Ф. Чихоткин, Р.К. Богданов, А.П. Закора. - М.: Изд-во ВНИИОЭНГ, 1997. 329 c.

12. A new method of combined rock drilling / T. Jiren, L. Yiyu, G. Zhaolong, X. Binwei, S. Huijuan et al. // International Journal of Mining Science and Technology. - 2014. - V. 24. - Iss. 1. P. 1-6.

13. Ивченко Г.И., Медведев Ю.И. Математическая статистика. М.: КД Либроком, 2014. - 352 с.

14. Sarle W.S. Neural Networks and Statistical Models // Proc. of the Nineteenth Annual SAS Users Group International Conference. Cary, NC, SAS Institute, April 3-4, 1994. - P. 1538-1550.

15. Syzrantseva K., Arishin V., Dvoynikov M. Optimization of the damping element of axial vibrations of the drilling string by com- puter simulation // Journal of Engineering and Applied Sciences. - 2016. - № 11 (10). - P. 2312-2315.

16. Application of hybrid artificial neural networks for predicting rate of penetration (ROP): a case study from Marun oil field / S.B. Ashrafi, M. Anemangely, M. Sabah, M.J. Ameri // Journal of Petroleum Science and Engineering. - 2019. - V. 175. P. 604-623.

17. Ahmed 0.S., Adeniran A.A., Samsuri A. Computational intelligence based prediction of drilling rate of penetration: a comparative study // Journal of Petroleum Science and Engineering. 2019. - № 172. - P. 1-12. DOI: 10.1016/j.petrol.2018.09.027.

18. A new methodology for optimization and prediction of rate of penetration during drilling operations / Y. Zhao, A. Noorbakhsh, M. Koopialipoor, A. Azizi, M.M. Tahir // Engineering with Computers. - 2019. - DOI: 10.1007/s00366-019-00715-2.

19. Hossain M.E., Al-Majed A.A. Fundamentals of Sustainable Drilling Engineering // Fundamentals of Sustainable Drilling Engineering. - 2015. - P. 785. D0I: 10.1002/9781119100300.

20. Soares C., Daigle H., Gray K. Evaluation of PDC bit ROP models and the effect of rock strength on model coefficients // Journal of Natural Gas Science and Engineering. - 2016. - № 34. P. 1225-1236. DOI: 10.1016/j.jngse.2016.08.012.

Поступила: 22.02.2019 2.

\section{Информация об авторах}

Tретьлк A.Я., доктор технических наук, профессор, академик РАЕН, заведующий кафедрой нефтегазовые техника и технологии Южно-Российского государственного политехнического университета (НПИ) им. М.И. Платова.

Кузнеиова A.B., кандидат технических наук, доцент, доцент кафедры программного обеспечения вычислительной техники Южно-Российского государственного политехнического университета (НПИ) им. М.И. Платова.

Борисов $\boldsymbol{K} . \boldsymbol{A}$., ассистент кафедры нефтегазовых техники и технологии Южно-Российского государственного политехнического университета (НПИ) им. М.И. Платова. 


\title{
DETERMINATION OF PDC CUTTER BREAKDOWNS USING REGRESSION AND NEURAL NETWORK MODELING
}

\author{
Alexander Ya. Tretyak', \\ 13050465@mail.ru
}

\author{
Alla V. Kuznetsova', \\ alvitkuz@yandex.ru
}

\author{
Konstantin A. Borisov', \\ 13020165@mail.ru
1 Platov South-Russian State Polytechnic University (NPI), 132, Prosveshcheniya street, Novocherkassk, 346428, Russia.

\begin{abstract}
The relevance. While drilling, the drill bits reinforced with PDC plates do not fully work out their life, as part of the cutting PDC elements fails due to their chipping, breakage or loss, which largely affects the final technical and economic indicators of well drilling. The aim of the research is to develop and to propose a neural network model to solve the problem of determining the percentage of breakage of cutting elements of drill bits reinforced with PDC, based on the available field data of drilling wells on rocks of VI-VIII category of drilling capacity.

Objects: the reasons causing failure of PDC plates on drill bits in well drilling in rocks of VI-VIII category of drilling capacity depending on the operating parameters of drilling.

Methods. The load on the bit, its speed and drilling speed were used as the determining factors for defining the percentage of breakdowns at constant values of the parameters of the washing liquid and bottom-hole assembly. Regression and neural network models of different configurations were used for data analysis.

Result. The analysis of regression models showed their unsuitability due to the nonlinear nature of the drilling speed at low pressures and high rpm. The authors proposed a two-stage neural network model in which the first neural network is used to determine the drilling speed, and the second - to predict the percentage of PDC plate breakdowns. Errors of the neural network ensemble on the test data do not exceed the values of the suitability of nonlinear models $-4,5 \%$ for the relative error and $12-15 \%$ of its maximum value. The proposed neural network model can be used in the development of technological regulations of working bits reinforced with PDC cutters.
\end{abstract}

\section{Key words:}

PDC breakdowns, artificial neural network, regression model, technological regulations of bit mining, torsional vibrations.

\section{REFERENCES}

1. Tretyak A.Ya., Popov V.V., Grossu A.N., Borisov K.A. Innovative approaches to designing highly efficient rock-breaking tool. GIAB, 2017, no. 8, pp. 225-230. In Rus.

2. Tretyak A.Ya., Chikhotkin V.F., Litkevich Yu.F., Aseeva A.E. Metod rascheta osevoy nagruzki i mekhanicheskoy skorosti bureniya dvukhyarusnogo dolota rezhushchego tipa D-2VV [The method of calculating the axial load and the mechanical speed of drilling a bunk bit of cutting type D-2VV]. Stroitelstvo neftyanykh i gazovykh skvazhin na sushe i na more, 2006, no. 3, pp. $13-18$.

3. Neskoromnykh V.V., Pushmin P.S. Metodika optimalnykh parametrov rezhima i usloviy bureniya skvazhin [Methods of optimal parameters and drilling conditions of wells]. Izvestiya Sibirskogo otdeleniya Sektsii nauk o Zemle RAEN, 2011, no. 1 (38), pp. 151-157.

4. Neskoromnykh V.V., Borisov K.I. Analytical study of the process of rock cutting-chipping with a chisel with PDC cutters. Bulletin of the Tomsk Polytechnic University, 2013, vol. 323, no. 1, pp. 191-195.

5. Tretyak A.A., Litkevich Yu.F., Aseeva A.E. Razrabotka metodiki rascheta narabotki porodorazrushayushchego instrumenta s almazno-tverdosplavnym vooruzheniem [Development of methodology for calculation of developments of rock cutting tool with a diamond-carbide armament]. Stroitelstvo neftyanykh $i$ gazovykh skvazhin na sushe i na more, 2010, no. 12, pp. 2-5.

6. Tretyak A.A. Vliyanie vibratsii na prochnostnye svoistva burovykh koronok, armirovannykh almazno-tverdosplavnymi plastinami [Influence of vibrations on the strength properties of drill bits reinforced with diamond-carbide plates]. Stroitelstvo neftyanykh $i$ gazovykh skvazhin na sushe $i$ na more, 2016, no. 10, pp. 20-24.

7. Sysoev N.I., Burenkov N.I., Tret'yak A.A. Calculating design parameters of PDC drill bits. Oil and Gas Journal Russia, 2012, no. 5 , pp. 56-58.

8. Simonov V.V., Yunin E.K. Vliyanie kolebatelnykh protsessov na rabotu burilnogo instrumenta [Influence of oscillatory processes on the operation of drilling tools]. Moscow, Nedra Publ., 1977. $210 \mathrm{p}$.

9. Vlasyuk V.I., Budyukov Yu.E., Spirin V.I. Tekhnicheskie sredstva i tekhnologii dlya povysheniya kachestva bureniya skvazhin [Technical means and technologies to improve the quality of drilling]. Tula, Grif i K Publ., 2013. 176 p.

10. Tre'yak A.A., Savenok 0.V., Shvets V.V. Burovye koronki, armirovannye almazno-tverdosplavnymi plastinami [Drill bits, reinforced with diamond-carbide plates] Novocherkassk, South Russian State Polytechnic University (NPI) Publ., 2015. 186 p.

11. Solovev N.V., Chikhotkin V.F., Bogdanov R.K., Zakora A.P. Resursosberegayushchaya tekhnologiya almaznogo bureniya $v$ slozhnykh geologicheskikh usloviyakh [Resource-saving technology of diamond drilling in complex geological conditions]. Moscow, VNIIOENG Publ., 1997. 329 p.

12. Jiren T., Yiyu L., Zhaolong G., Binwei X., Huijuan S. A new method of combined rock drilling. International Journal of $\mathrm{Mi}$ ning Science and Technology, 2014, vol. 24, Iss. 1, pp. 1-6.

13. Ivchenko G.I., Medvedev Yu.I. Matematicheskaya statistika [Mathematical statistics]. Moscow, KD Librokom Publ., 2014. $352 \mathrm{p}$. 
14. Sarle W.S. Neural Networks and Statistical Models. Proc. of the Nineteenth Annual SAS Users Group International Conference. Cary, NC, SAS Institute, April 3-4, 1994. pp. 1538-1550.

15. Syzrantseva K., Arishin V., Dvoynikov M. Optimization of the damping element of axial vibrations of the drilling string by computer simulation. Journal of Engineering and Applied Sciences, 2016, no. 11 (10), pp. 2312-2315.

16. Ashrafi S.B., Anemangely M., Sabah M., Ameri M.J. Application of hybrid artificial neural networks for predicting rate of penetration (ROP): a case study from Marun oil field. Journal of Petroleum Science and Engineering, 2019, vol. 175, pp. 604-623.

17. Ahmed 0.S., Adeniran A.A., Samsuri A. Computational intelligence based prediction of drilling rate of penetration: a comparative study. Journal of Petroleum Science and Engineering, 2019, no. 172, pp. 1-12. D0I: 10.1016/j.petrol.2018.09.027.
18. Zhao Y., Noorbakhsh A., Koopialipoor M., Azizi A., Tahir M.M. A new methodology for optimization and prediction of rate of penetration during drilling operations. Engineering with Computers, 2019. D0I: $10.1007 / \mathrm{s} 00366-019-00715-2$.

19. Hossain M.E., Al-Majed A.A. Fundamentals of Sustainable Drilling Engineering. Fundamentals of Sustainable Drilling Engineering, 2015. 785 p. DOI: $10.1002 / 9781119100300$.

20. Soares, C., Daigle, H., Gray, K. Evaluation of PDC bit ROP models and the effect of rock strength on model coefficients. Journal of Natural Gas Science and Engineering, 2016, no. 34, pp. 1225-1236. D0I: 10.1016/j.jngse.2016.08.012.

Received: 22 February 2019.

\section{Informstion about the authors}

Alexander Ya. Tretyak, Dr. Sc., professor, Academician of Russian Academy of Natural Sciences, Platov SouthRussian State Polytechnic University (NPI).

Alla V. Kuznetsova, Cand Sc., associate professor, Platov South-Russian State Polytechnic University (NPI).

Konstantin A. Borisov, assistant, Platov South-Russian State Polytechnic University (NPI). 